\title{
Photo-Induced Terminal Hydride of [FeFe]-Hydrogenase Biomimetic Complexes
}

\author{
Shuqiang Niu, ${ }^{\dagger}$ Anne E. Nelson,,${ }^{\ddagger}$ Patricia De La Torre, ${ }^{\ddagger}$ Haixia Li,${ }^{\dagger}$ Carmen F. Works, ${ }^{* *}$ \\ and Michael B. Hall ${ }^{\dagger *}$ \\ ${ }^{\dagger}$ Department of Chemistry, Texas A\&M University, College Station, TX 77843-3255 \\ Department of Chemistry, Sonoma State University, Rohnert Park, California 94928
}

*To whom correspondence may be addressed. E-mail:

\section{Computational Details}

Spin-unrestricted DFT calculations with broken-symmetry (BS) molecular orbital (MO) wavefunctions ${ }^{1}$ were performed for geometries and energies of ground and excited states of $\left[\mathrm{Fe}_{2}(\mu-\mathrm{pdt})(\mathrm{CO})_{4}\left(\mathrm{PMe}_{3}\right)_{2}\right]^{0}(\mathbf{2})$ and $\left[\mathrm{Fe}_{2}(\mu-\mathrm{pdt})(\mu-\mathrm{H})(\mathrm{CO})_{4}\left(\mathrm{PMe}_{3}\right)_{2}\right]^{+}\left(\mathbf{2} \mathbf{H}^{+}\right)$along possible photochemical reaction pathways. The geometry optimizations and energy calculations were carried out using $\mathrm{NWChem}^{2}$ implementation of M06 density functional, ${ }^{3}$ using Dgauss DZVP2 basis sets, ${ }^{4}$ and using extra fine grids for energies to target accuracy of $10^{-8}$ au..$^{2}$ This calculational approach was tested in benchmark studies for a balance of accuracy and efficiency in the descriptions of structural and energetic properties of $[\mathrm{NiFe}]-$ and $[\mathrm{FeFe}]-\mathrm{H}_{2}$ ase active sites (see Supporting Information). Photochemical products may have open-shell spin-coupled electronic structures, but the calculated BS state energies for them are weighted averages of pure spin states instead of an energy for a pure state. ${ }^{5}$ Thus, Yamaguchi's approximate spin projection (AP) procedure was implemented for energy corrections of some important cases. ${ }^{6}$ The solvation free energies are calculated by using the SMD continuum solvation model ${ }^{7}$ for optimized geometries in the gas phase and are included in the energetic analysis. Transition states (TS) were optimized by an eigenvalue following optimization method, in which the final updated Hessian has only one negative eigenvalue with eigenvectors 
representing the rotation of terminal ligands for isomerization. Although the timedependent DFT (TD-DFT) calculations can give very good descriptions of excited states, and corresponding bond dissociation/formation and optical properties in photochemistry, due to complexity of electronic structure of excited states of $[\mathrm{FeFe}]-\mathrm{H}_{2}$ ase analogues, it is difficult to completely find out those possible photoproducts at UV/visible light energy by the TD-DFT adiabatic approximation. For instance, the species with high-spin iron sites and the $\mathrm{PMe}_{3}$ dissociated species were roughly excluded on the basis of the band assignments and the excited state geometry optimizations by TD-DFT investigations. ${ }^{8}$ Therefore, for IR spectra characterization, we carry out BS-DFT calculations directly on the species along possible photochemical reaction pathways, including ligand isomerization, $\mathrm{CO}$ dissociation, $\mathrm{PMe}_{3}$ dissociation, $\mathrm{Fe}-\mathrm{S}$ cleavage, and $\mathrm{CO}$ dissociation followed by electron-donor ligand/solvent substitution.

Vibrational frequency calculations were performed on the optimized structures of all ground and excited states at the M06/DZVP2 level for simulations of infrared spectra of photochemical and thermal processes of the $\left[\mathrm{Fe}_{2}(\mu-\mathrm{pdt})(\mathrm{CO})_{4}\left(\mathrm{PMe}_{3}\right)_{2}\right]^{0}$ complex 2 and $\left[\mathrm{Fe}_{2}(\mu-\mathrm{pdt})(\mu-\mathrm{H})(\mathrm{CO})_{4}\left(\mathrm{PMe}_{3}\right)_{2}\right]^{+} \mathbf{2} \mathbf{H}^{+}$, in which the $\mathrm{CO}$ vibrational frequencies were obtained from the projected hessian. ${ }^{2}$ To better match experimental vibrational frequencies, the $\mathrm{CO}$ vibrational frequencies produced by DFT calculations at the M06/DZVP2 level were scaled by a factor of 0.9432 , which was obtained by a correlation of the calculated $\mathrm{CO}$ vibrational frequency with our experimental value of the largest parent peak of the $\left[\mathrm{Fe}_{2}(\square \text {-pdt })(\mathrm{CO})_{4}\left(\mathrm{PMe}_{3}\right)_{2}\right]^{0}$ complex at $1942 \mathrm{~cm}^{-1}$ in toluene. Although solvent effects may lead to redshifts of $\mathrm{CO}$ vibrational frequencies of $\left[\mathrm{Fe}_{2}(\mu-\right.$ pdt $\left.(\mathrm{CO})_{4}\left(\mathrm{PMe}_{3}\right)_{2}\right]^{0}$ with the increase of solvent dielectric constant (or dipole moment), 
the systematic errors in vibrational frequency calculations based on the optimized geometries in the gas phase can be corrected by the optimized scale factor (Table S4).

The MO and IR spectra visualizations were performed using the Ampac Graphical User Interface (AGUI) program. ${ }^{9}$ Computational IR spectra were generated by Microsoft Office Excel program based on the calculated vibrational frequencies and intensities.

\section{Refernces}

(1) Cramer, C. J.; Truhlar, D. G., Density functional theory for transition metals and transition metal chemistry. Phys Chem Chem Phys 2009, 11, 10757-10816.

(2) Valiev, M.; Bylaska, E. J.; Govind, N.; Kowalski, K.; Straatsma, T. P.; Van Dam, H. J. J.; Wang, D.; Nieplocha, J.; Apra, E.; Windus, T. L.; de Jong, W., NWChem: A comprehensive and scalable open-source solution for large scale molecular simulations. Comput. Phys. Commun. 2010, 181, 1477-1489.

(3) Cramer, C. J., Essentials of computational chemistry : theories and models. 2nd ed.; Wiley: Chichester, West Sussex, England ; Hoboken, NJ, 2004; p xx, 596 p.

(4) Godbout, N.; Salahub, D. R.; Andzelm, J.; Wimmer, E., Optimization of Gaussian-Type Basis-Sets for Local Spin-Density Functional Calculations .1. Boron Through Neon, Optimization Technique and Validation. Can. J. Chem. 1992, 70, 560 571.

(5) Noodleman, L.; Peng, C. Y.; Case, D. A.; Mouesca, J. M., Orbital interactions, electron delocalization and spin coupling in iron-sulfur clusters. Coord. Chem. Rev. 1995, 144, 199-244.

(6) Kitagawa, Y.; Saito, T.; Ito, M.; Shoji, M.; Koizumi, K.; Yamanaka, S.;

Kawakami, T.; Okumura, M.; Yamaguchi, K., Approximately spin-projected geometry optimization method and its application to di-chromium systems. Chem. Phys. Lett. 2007, 442, 445-450.

(7) Marenich, A. V.; Cramer, C. J.; Truhlar, D. G., Universal solvation model based on solute electron density and on a continuum model of the solvent defined by the bulk dielectric constant and atomic surface tensions. J. Phys. Chem. B 2009, 113, 6378-6396. (8) Bertini, L.; Alberto, M. E.; Arrigoni, F.; Vertemara, J.; Fantucci, P.; Bruschi, M.; Zampella, G.; De Gioia, L., On the photochemistry of $\mathrm{Fe}_{2}(\mathrm{edt})(\mathrm{CO})_{4}\left(\mathrm{PMe}_{3}\right)_{2}$, a FeFe hydrogenase model: A DFT/TDDFT investigation. Int. J. Quantum Chem. 2018, 118, e25537.

(9) Glaser, R., AMPAC 9. J. Am. Chem. Soc. 2009, 131, 13564-13564. 
Table S1. The Experimental and M06/DZVP2 Calculated Relative Energies $\Delta \mathrm{E}$ $(\mathrm{kcal} / \mathrm{mol})$ in the DCM, CO vibrational Frequencies $\left(\mathrm{cm}^{-1}\right)$ of the Thermal and Photochemical Isomers $1 \mathrm{H}^{+}$.

\begin{tabular}{|c|c|c|c|c|c|c|}
\hline & $\Delta \mathrm{E}$ & $v^{1} \mathrm{CO}$ & $v^{2} \mathrm{CO}$ & $v^{3} \mathrm{CO}$ & $v^{4} \mathrm{CO}$ & Intensity \\
\hline $1 \mathbf{a H}_{\mathrm{b}}{ }^{+}$ & 0.00 & 1995 & 1997 & 2019 & 2037 & $\mathrm{~m}, \mathrm{~m}, \mathrm{~s}, \mathrm{vw}$ \\
\hline $1 \mathrm{bH}_{\mathrm{b}}{ }^{+}$ & 3.58 & 2000 & 2004 & 2029 & 2048 & $\mathrm{w}, \mathrm{m}, \mathrm{m}, \mathrm{m}$ \\
\hline $1 \mathrm{cH}_{\mathbf{b}}{ }^{+}$ & 3.40 & 2002 & 2006 & 2030 & 2048 & $\mathrm{~m}, \mathrm{~m}, \mathrm{~m}, \mathrm{~m}$ \\
\hline $\mathbf{1 d H _ { b }}{ }^{+}$ & 4.44 & 1999 & 2005 & 2034 & 2048 & $\mathrm{~m}, \mathrm{~m}, \mathrm{~m}, \mathrm{~m}$ \\
\hline $1 \mathrm{eH}_{\mathrm{b}}{ }^{+}$ & 7.69 & 2013 & 2022 & 2038 & 2055 & $\mathrm{vw}, \mathrm{s}, \mathrm{w}, \mathrm{m}$ \\
\hline $1 \mathrm{a} 2 \mathrm{H}_{\mathbf{t}}^{+}$ & 20.10 & 1917 & 2011 & 2013 & 2052 & $\mathrm{w}, \mathrm{w}, \mathrm{s}, \mathrm{m}$ \\
\hline $1 \mathrm{a} 3 \mathrm{H}_{\mathrm{t}}{ }^{+}$ & 17.98 & 1920 & 2011 & 2040 & 2051 & $\mathrm{w}, \mathrm{m}, \mathrm{m}, \mathrm{m}$ \\
\hline $1 \mathrm{a} 4 \mathrm{H}_{\mathrm{b}}{ }^{+}$ & 30.35 & 1981 & 2018 & 2037 & 2050 & $\mathrm{~m}, \mathrm{~m}, \mathrm{~s}, \mathrm{~W}$ \\
\hline $1 \mathrm{a5} \mathrm{H}_{\mathrm{t}}^{+}$ & 47.47 & 1888 & 2025 & 2036 & 2053 & $\mathrm{vw}, \mathrm{w}, \mathrm{m}, \mathrm{m}$ \\
\hline $1 \mathrm{a} 5 \mathrm{bH}_{\mathrm{t}}^{+}$ & 53.43 & 1904 & 2016 & 2028 & 2053 & $\mathrm{w}, \mathrm{w}, \mathrm{m}, \mathrm{m}$ \\
\hline $1 \mathrm{c} 2 \mathrm{H}_{\mathrm{t}}^{+}$ & 24.60 & 1951 & 2019 & 2027 & 2052 & $\mathrm{w}, \mathrm{w}, \mathrm{m}, \mathrm{m}$ \\
\hline $1 \mathrm{c} 3 \mathrm{H}_{\mathrm{t}}{ }^{+}$ & 18.47 & 1948 & 2014 & 2036 & 2048 & $\mathrm{w}, \mathrm{m}, \mathrm{m}, \mathrm{m}$ \\
\hline $1 \mathrm{c} 4 \mathrm{H}_{\mathrm{t}}^{+}$ & 17.20 & 1889 & 2010 & 2016 & 2041 & $\mathrm{~m}, \mathrm{~m}, \mathrm{~m}, \mathrm{w}$ \\
\hline $1 \mathrm{c} 5 \mathrm{H}_{\mathrm{t}}^{+}$ & 20.09 & 1915 & 2007 & 2013 & 2044 & $\mathrm{~m}, \mathrm{~m}, \mathrm{~m}, \mathrm{~m}$ \\
\hline $1 \mathrm{abH}_{\mathrm{s}}{ }^{+}$ & 30.56 & 1943 & 1958 & 1991 & 2004 & $\mathrm{~m}, \mathrm{~m}, \mathrm{~s}, \mathrm{vw}$ \\
\hline $1 \mathrm{c} 6 \mathrm{H}_{\mathrm{S}}{ }^{+}$ & 28.89 & 1956 & 1974 & 1988 & 2018 & $\mathrm{~m}, \mathrm{w}, \mathrm{s}, \mathrm{m}$ \\
\hline $1 \mathrm{a} 7 \mathrm{H}_{\mathrm{S}}{ }^{+}$ & 29.84 & 1958 & 1966 & 1986 & 2007 & $\mathrm{w}, \mathrm{m}, \mathrm{s}, \mathrm{m}$ \\
\hline $1 \mathrm{aT} 7 \mathrm{H}_{\mathrm{S}}{ }^{+}$ & 40.90 & 1954 & 1961 & 1997 & 2006 & $\mathrm{~m}, \mathrm{~s}, \mathrm{~m}, \mathrm{~m}$ \\
\hline $1 \mathrm{cT} 7 \mathrm{H}_{\mathrm{S}}{ }^{+}$ & 44.81 & 1957 & 1977 & 1995 & 2015 & $\mathrm{~m}, \mathrm{~m}, \mathrm{~m}, \mathrm{~m}$ \\
\hline $1 \mathrm{cT} 8 \mathrm{H}_{\mathrm{S}}{ }^{+}$ & 53.68 & 1922 & 1963 & 1990 & 2004 & $\mathrm{~s}, \mathrm{~m}, \mathrm{~s}, \mathrm{~W}$ \\
\hline Exp./DCM & & & 1990 & 2032 & 2046 & $\mathrm{~m}, \mathrm{~m}, \mathrm{~s}, \mathrm{vw}$ \\
\hline Exp./Acetone & & & 1989 & 2031 & & \\
\hline Exp./MeCN & & & 1990 & 2030 & & \\
\hline $\operatorname{Exp} . / \operatorname{DCM}\left(\mathbf{N}_{2}\right)^{\mathrm{a}}$ & & 1944 & 1977 & 2017 & $2050^{b}$ & \\
\hline $\operatorname{Exp} . / D C M(C O)^{a}$ & & 1940 & 1972 & 2019 & $2048^{b}$ & \\
\hline $\operatorname{Exp} . /$ Acetone $\left(\mathbf{N}_{2}\right)^{\mathrm{a}}$ & & 1944 & 1977 & 2015 & $2048^{b}$ & \\
\hline $\operatorname{Exp}$ /Acetone $(\mathrm{CO})^{\mathrm{a}}$ & & 1943 & 1972 & 2011 & NA & \\
\hline
\end{tabular}

${ }^{\mathrm{a}}$ Under irradiation at a minute time scale. ${ }^{\mathrm{b}}$ Difficult to observe in difference spectra 
Table S2. The M06/DZVP2 Calculated Relative Energies $\Delta \mathrm{E}(\mathrm{kcal} / \mathrm{mol})$ in DCM, CO vibrational Frequencies $\left(\mathrm{cm}^{-1}\right)$ of Photo-Products of the CO Loss of $\mathbf{1 a H} \mathbf{H}^{+}$.

\begin{tabular}{|c|c|c|c|c|c|}
\hline & $\Delta \mathrm{E}^{\mathrm{a}}$ & $v^{1} \mathrm{CO}$ & $v^{2} \mathrm{CO}$ & $v^{3} \mathrm{CO}$ & Intensity \\
\hline $2 \mathrm{a} 2 \mathrm{H}_{\mathrm{b}}{ }^{+}$ & 52.82 & 1998 & 2002 & 2032 & m.m.w \\
\hline $2 \mathrm{a} 4 \mathrm{H}_{\mathrm{b}}{ }^{+}$ & 50.80 & 1997 & 1998 & 2029 & $\mathrm{~m}, \mathrm{~m}, \mathrm{w}$ \\
\hline $2 \mathrm{a} 3 \mathrm{aH}_{\mathrm{t}}^{+}$ & 76.26 & 1992 & 2000 & 2029 & $\mathrm{vw}, \mathrm{s}, \mathrm{m}$ \\
\hline $2 \mathrm{a} 3 \mathrm{bH} \mathrm{t}^{+}$ & 68.57 & 1884 & 2010 & 2044 & $\mathrm{w}, \mathrm{m}, \mathrm{m}$ \\
\hline $2 \mathrm{a} 3 \mathrm{cH}_{\mathrm{t}}^{+}$ & 72.00 & 1882 & 2025 & 2040 & $\mathrm{w}, \mathrm{m}, \mathrm{m}$ \\
\hline $2 \mathrm{a3dH}_{\mathrm{t}}^{+}$ & 64.79 & 1922 & 1996 & 2043 & $\mathrm{~m}, \mathrm{~m}, \mathrm{~m}$ \\
\hline $2 \mathrm{a}^{2} \mathrm{aH}_{\mathrm{b}}{ }^{+}$ & 77.27 & 1969 & 2021 & 2050 & $\mathrm{~m}, \mathrm{~m}, \mathrm{~m}$ \\
\hline $2 \mathrm{a}^{2} \mathrm{bH}_{\mathrm{b}}^{+}$ & 55.03 & 1997 & 2013 & 2043 & $\mathrm{~m}, \mathrm{~m}, \mathrm{~m}$ \\
\hline $2 \mathrm{a} 6 \mathrm{H}_{\mathbf{t}}^{+}$ & 62.63 & 1973 & 2003 & 2038 & $\mathrm{w}, \mathrm{m}, \mathrm{m}$ \\
\hline $2 \mathrm{aT} 2 \mathrm{H}_{\mathrm{b}}{ }^{+}$ & 43.06 & 2000 & 2005 & 2037 & $\mathrm{~m}, \mathrm{~m}, \mathrm{~m}$ \\
\hline $2 \mathrm{aT} 4 \mathrm{H}_{\mathrm{b}}^{+}$ & 40.22 & 2005 & 2006 & 2036 & $\mathrm{~m}, \mathrm{~m}, \mathrm{w}$ \\
\hline $2 \mathrm{aT} \mathrm{bH}_{\mathrm{t}}^{+}$ & 57.43 & 1887 & 2015 & 2049 & $\mathrm{w}, \mathrm{m}, \mathrm{m}$ \\
\hline $2 \mathrm{aT3} \mathrm{cH}_{\mathrm{t}}^{+}$ & 56.61 & 1940 & 2027 & 2040 & $\mathrm{w}, \mathrm{m}, \mathrm{m}$ \\
\hline $2 \operatorname{aT} \mathrm{dH}_{\mathrm{t}}^{+}$ & 58.95 & 1907 & 2001 & 2040 & $\mathrm{~m}, \mathrm{~m}, \mathrm{~m}$ \\
\hline $2 \mathrm{aT}^{2} \mathrm{aH}_{\mathrm{b}}{ }^{+}$ & 90.29 & 1986 & 2018 & 2077 & $\mathrm{~m}, \mathrm{~m}, \mathrm{w}$ \\
\hline
\end{tabular}

a In DCM. 
Table S3. The M06/DZVP2 Calculated Relative Energies $\Delta \mathrm{E}$ ( $\mathrm{kcal} / \mathrm{mol})$, CO vibrational Frequencies $\left(\mathrm{cm}^{-1}\right)$ of $\mathrm{PMe}_{3}$ Photolysis Products of $\mathbf{1 a H}$.

\begin{tabular}{lrrrrrr}
\hline & $\Delta \mathrm{E}^{\mathrm{a}}$ & $v^{1} \mathrm{CO}$ & $v^{2} \mathrm{CO}$ & $v^{3} \mathrm{CO}$ & $v^{4} \mathrm{CO}$ & Intensity \\
\hline $\mathbf{3 a H}_{\mathbf{b}}{ }^{+}$ & 56.39 & 2018 & 2040 & 2048 & 2069 & $\mathrm{~m}, \mathrm{~m}, \mathrm{~s}, \mathrm{w}$ \\
$\mathbf{3 a T H}_{\mathbf{b}}{ }^{+}$ & 57.39 & 2017 & 2047 & 2049 & 2096 & $\mathrm{~m}, \mathrm{~s}, \mathrm{w}, \mathrm{m}$ \\
$\mathbf{3 a 2 H}_{\mathbf{b}}{ }^{+}$ & 82.42 & 2019 & 2036 & 2059 & 2070 & $\mathrm{~m}, \mathrm{~m}, \mathrm{~s}, \mathrm{w}$ \\
$\mathbf{3 a 2 b H}_{\mathbf{b}}{ }^{+}$ & 60.07 & 2026 & 2042 & 2055 & 2075 & $\mathrm{~m}, \mathrm{~m}, \mathrm{~m}, \mathrm{~m}$ \\
$\mathbf{3 a 3 H}_{\mathbf{t}}{ }^{+}$ & 71.20 & 1957 & 2045 & 2050 & 2080 & $\mathrm{w}, \mathrm{m}, \mathrm{m}, \mathrm{m}$ \\
$\mathbf{3 a 3 T H}_{\mathbf{t}}{ }^{+}$ & 74.36 & 1969 & 2046 & 2068 & 2099 & $\mathrm{w}, \mathrm{m}, \mathrm{m}, \mathrm{m}$ \\
$\mathbf{3 a 3 b H}_{\mathbf{t}}{ }^{+}$ & 74.95 & 1943 & 2027 & 2057 & 2072 & $\mathrm{~m}, \mathrm{~m}, \mathrm{~m}, \mathrm{~m}$ \\
$\mathbf{3 a 3 b T H}_{\mathbf{t}}{ }^{+}$ & 74.64 & 1952 & 2025 & 2061 & 2095 & $\mathrm{w}, \mathrm{m}, \mathrm{m}, \mathrm{m}$ \\
$\mathbf{3 a 4 H}_{\mathbf{t}}{ }^{+}$ & 77.14 & 1991 & 2047 & 2059 & 2090 & $\mathrm{w}, \mathrm{m}, \mathrm{m}, \mathrm{m}$ \\
\hline
\end{tabular}

a In DCM. 
Table S4. The Experimental and M06/DZVP2 Calculated CO Vibrational

Frequencies $\left(\mathrm{cm}^{-1}\right)$ in the Solution.

\begin{tabular}{llllllll}
\hline & & factor & $v^{1} \mathrm{CO}$ & $v^{2} \mathrm{CO}$ & $v^{3} \mathrm{CO}$ & $v^{4} \mathrm{CO}$ & Intensity \\
\hline 2a & Cal. & 0.0000 & 2012 & 2023 & 2059 & 2073 & $\mathrm{~m}, \mathrm{~m}, \mathrm{~s}, \mathrm{vw}$ \\
& Scaled & 0.9432 & 1898 & 1908 & 1942 & 1955 & $\mathrm{~m}, \mathrm{~m}, \mathrm{~s}, \mathrm{vw}$ \\
& Exp. $^{\mathrm{a}}$ & & 1900 & 1900 & 1942 & & $\mathrm{~m}, \mathrm{~m}, \mathrm{~s}, \mathrm{vw}$ \\
& Exp. $^{\mathrm{b}}$ & & & 1900 & 1942 & & $\mathrm{~m}, \mathrm{~s}$ \\
\hline
\end{tabular}

${ }^{\mathrm{a}}$ In toluene. ${ }^{\mathrm{b}}$ In $\mathrm{MeCN}$. 
Scheme S1
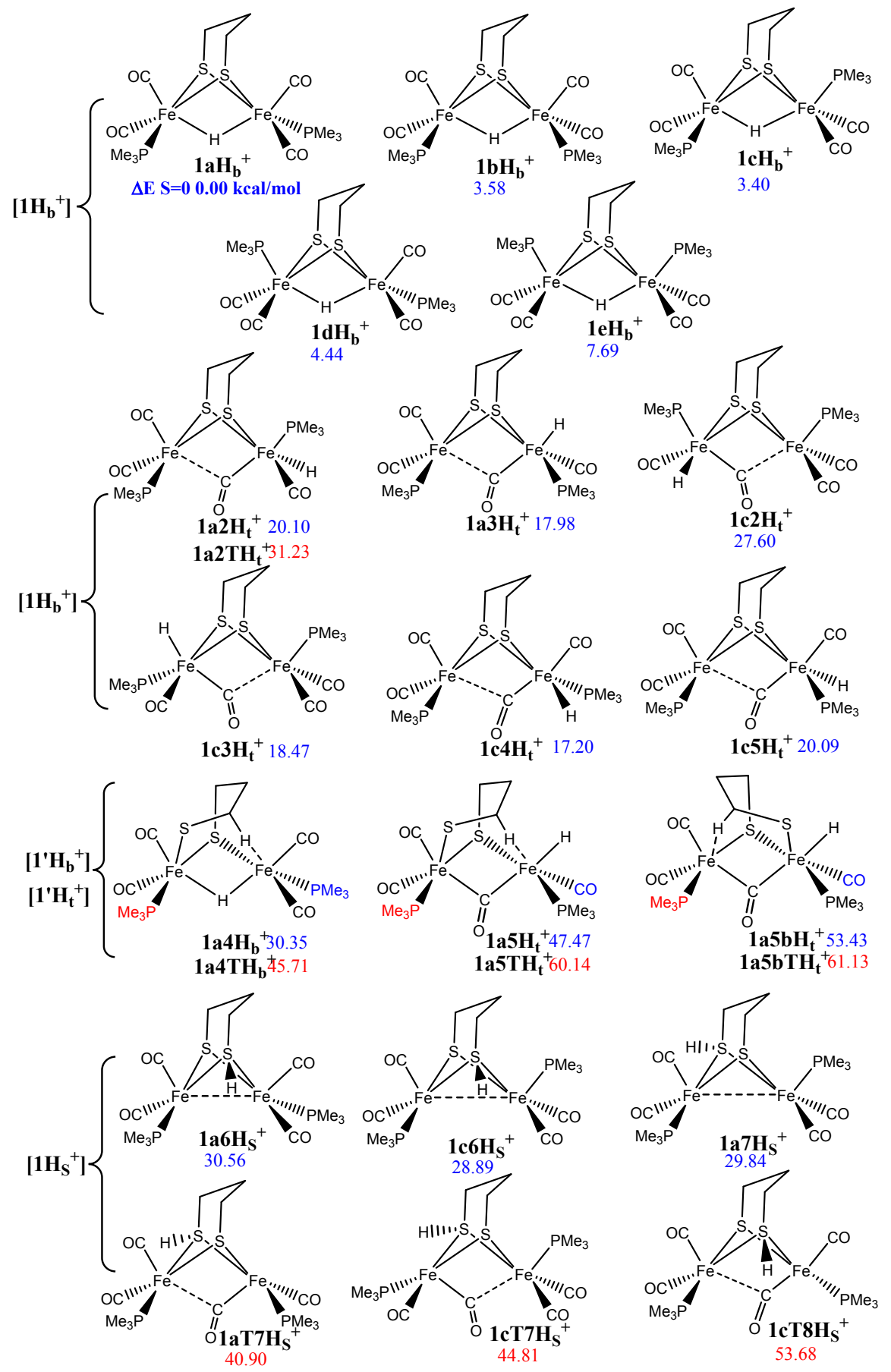
Scheme S2

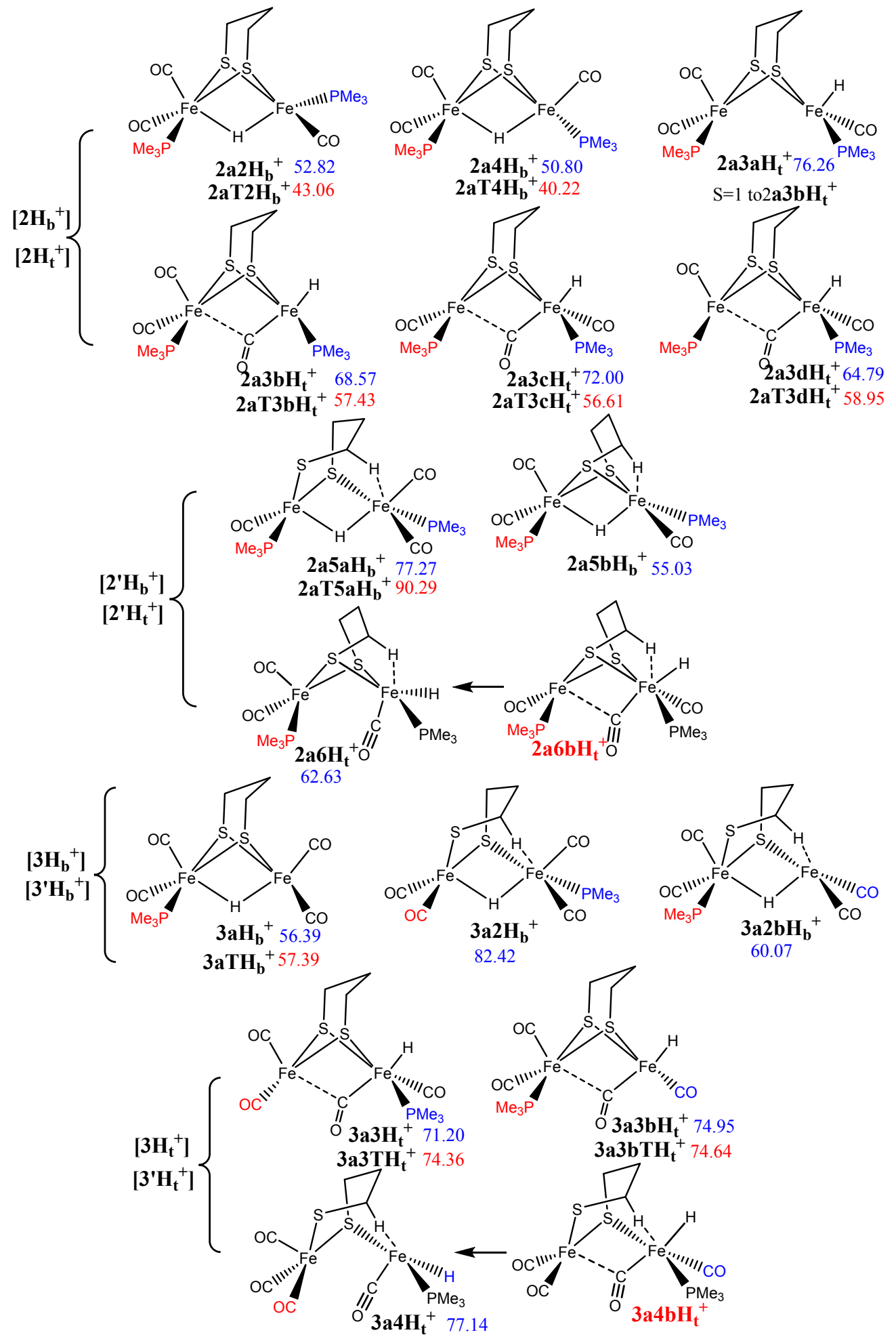



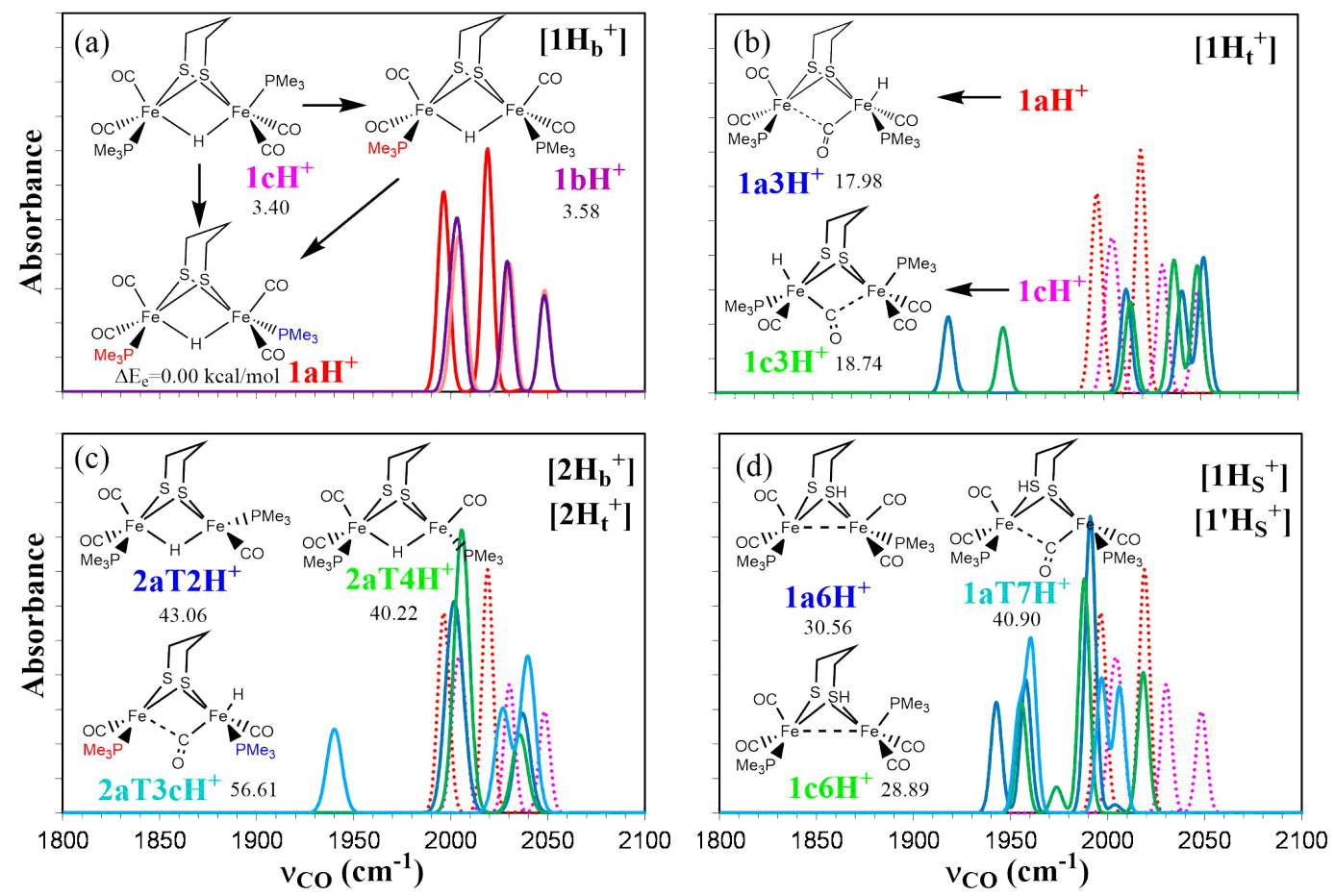

Figure S1. DFT simulated IR spectrum and relative energies $\Delta \mathrm{E}_{\mathrm{e}}$ (in DCM, kcal $/ \mathrm{mol}$ ) of the $\mathbf{1 H}^{+}$and possible photochemical products: (a) the parent $\left[\mathbf{1 H}_{\mathbf{b}}{ }^{+}\right]$isomers, $\mathbf{1 a H}^{+}$(red), $\mathbf{1} \mathbf{c H}^{+}$(pink) and $\mathbf{1} \mathbf{d H}^{+}$(purple); (b) the hydride rearrangement isomers with a terminal hydride $\left[\mathbf{1 H}_{\mathbf{t}}^{+}\right]$(the parent peaks in dash line); (c) the CO loss isomers $\left[\mathbf{2 H}_{\mathbf{b}}{ }^{+}\right]$and $\left[\mathbf{2} \mathbf{H}_{\mathbf{t}}^{+}\right]$; (d) the protonated-pdt isomers $\left[\mathbf{1 H}_{\mathbf{s}}{ }^{+}\right]$and $\left[\mathbf{1}^{\prime} \mathbf{H}_{\mathbf{S}}{ }^{+}\right]$. 


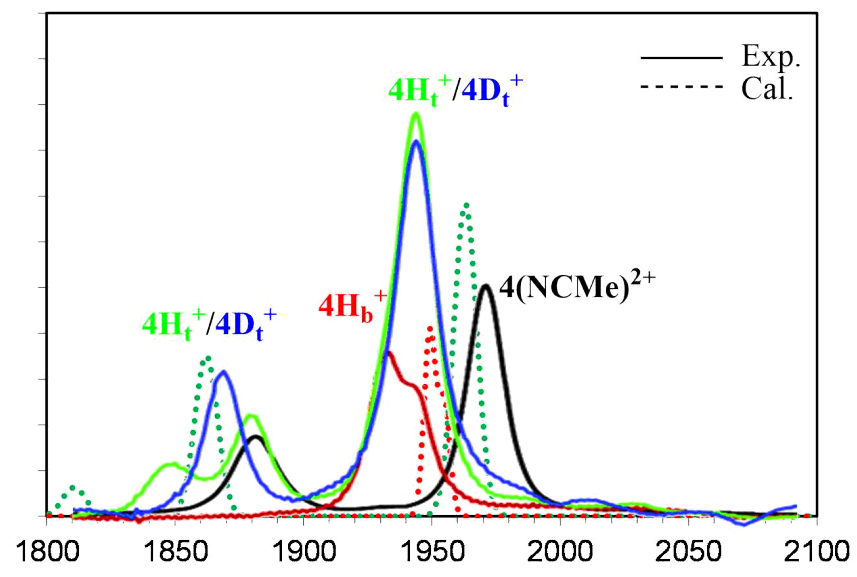

Figure S2. Experimental (solid line) and DFT simulated IR spectrum (dash line) of the $\mathbf{4} \mathbf{H}_{\mathbf{b}}{ }^{+}$(red) and $\mathbf{4} \mathbf{H}_{\mathbf{t}}^{+}$(green) isomers. 


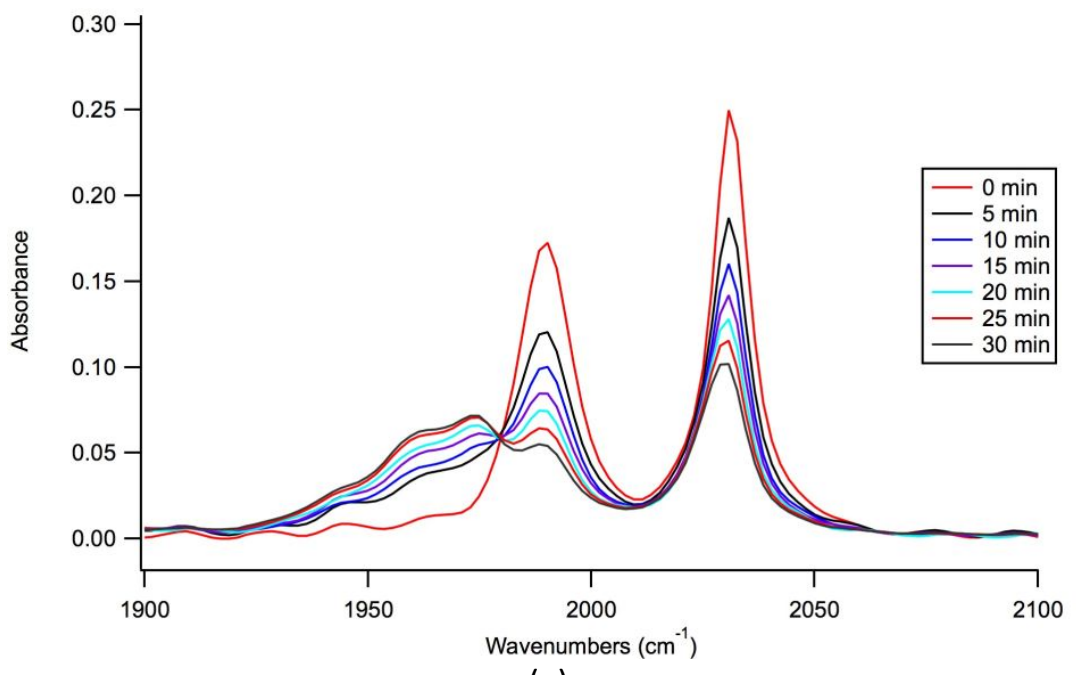

(a)

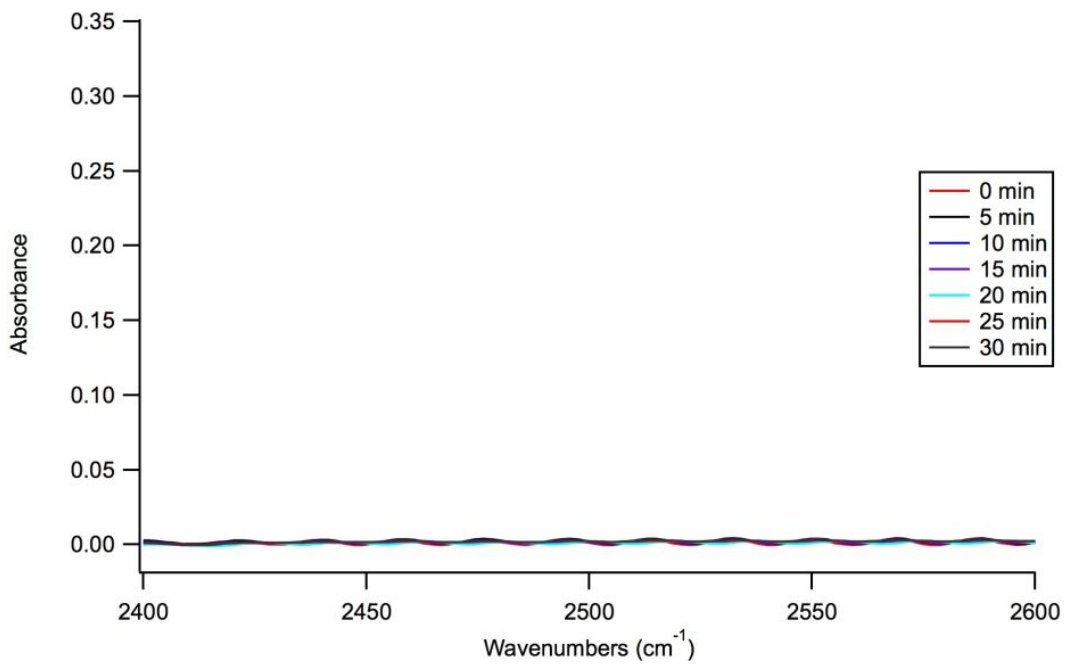

(b)

Figure S3. IR spectral changes of $\mathbf{1 H}^{+}$upon photolysis with $365 \mathrm{~nm}$ light in acetonitrile in the $\mathrm{CO}$ vibrational region (a) and the $\mathrm{S}-\mathrm{H}$ vibrational region (b). 